\title{
Hanseníase no município de Buriticupu, Estado do Maranhão: busca ativa na população estudantil
}

\author{
Leprosy in the municipality of Buriticupu, State of Maranhão: active \\ search among the student population
}

\author{
Antonio Rafael da Silva ${ }^{1}$, Édson Gustavo Lima Portela ${ }^{1}$, Wilma Batista de Matos ${ }^{1}$, \\ Camila Cristina Bastos Silva ${ }^{1}$ e Eloisa da Graça do Rosario Gonçalves ${ }^{1}$
}

\begin{abstract}
RESUMO
Oestudo teve o objetivo de determinar o coeficiente de detecção da hanseníase na população estudantil do município de Buriticupu, Estado do Maranhão. Empregou-se o método de busca ativa de casos, abrangendo-se uma população de 14.653 estudantes, em 53 escolas da rede de ensino municipal. O exame clínico definiu o diagnóstico de hanseníase em 20 estudantes, o que representa um coeficiente de detecção de 13,6/10.000 estudantes. Em todos foi realizada baciloscopia de linfa cutânea, a qual foi positiva em 2 amostras. A forma clínica predominante foi a indeterminada com 12 (60\%) casos, seguida da tuberculóide com 5 (25\%) e da dimorfa com 3 (15\%). Procedeu-se a realização de biópsia da lesão em 11 pacientes, tendo o estudo histopatológico revelado achados comuns de hiperceratose, infiltrado inflamatório mononuclear, vasos ectásicos e músculo eretor de pêlos, isolado. O estudo permitiu, ainda, a identificação de outras doenças infecciosas da pele: pitiríase versicolor em 793, escabiose em 361 e dermatofitoses em 119 indivíduos. Condições diversas e inespecíficas como cicatrizes, nevos e escoriações foram detectadas em 1.020 estudantes.
\end{abstract}

Palavras-chaves: Hanseníase. Prevalência. Busca ativa. Maranhão.

\section{ABSTRACT}

This study had the aim of determining the coefficient of leprosy detection among the student population of the municipality of Buriticupu, State of Maranhão. An active case search method was used, covering a population of 14,653 students, in 53 schools within the municipal network. Clinical examination gave rise to a leprosy diagnosis for 20 students, which represents a detection coefficient of 13.6/10,000 students. Cutaneous lymph bacilloscopy was performed on all cases, and it was positive in two samples. The predominant clinical form was the indeterminate form, with 12 cases (60\%), followed by the tuberculoid form, with five (25\%) and the dimorphous form with two (10\%). Biopsies were then taken from the lesions of 11 patients, and histopathological analysis showed common findings of hyperkeratosis, mononuclear inflammatory infiltrate and ectasia of vessels and isolated arrector pili muscles. The study also enabled identification of some other infectious skin diseases: pityriasis versicolor in 793 , scabies in 361 and dermatophytosis in 119. A variety of nonspecific conditions were also detected, such as scars, nevi and excoriation, in 1,020 students.

Key-words: Leprosy. Prevalence. Active search. Maranhão.

A hanseníase continua como um problema muito importante de saúde pública no Brasil, responsável por $94 \%$ dos casos novos detectados nas Américas ${ }^{1}$.

0 coeficiente de prevalência da doença, que era de 10,4 casos/10.000 habitantes, em 1985, apresentou redução progressiva ao longo da década de 1990. No início de 2004, o indicador era de 4,6/10.000, menor apenas que os coeficientes das Ilhas Marshall e dos Estados Federados da Micronésia 7 . A partir desse ano, com a adoção da prevalência de ponto como indicador da magnitude da doença no país, houve uma acentuação da tendência, chegando-se ao valor de 1,48/10.000 em 20055. A situação da doença, no entanto, não é homogênea, com as regiões mostrando tendências distintas em relação à prevalência e controle. A meta estabelecida de reduzir a prevalência para menos de 1/10.000 no ano 2000 (prazo posteriormente adiado para 2005) só foi alcançada pelos estados de Santa Catarina e Rio Grande do Sul ${ }^{3}$.

A detecção de casos novos permaneceu elevada nesse tempo, registrando-se 38.410 casos (coeficiente de detecção de 2,09/10.000), no ano de 2005. Além disso, os dados do Ministério da Saúde revelam elevada ocorrência de casos novos entre menores de 15 anos, especialmente nas regiões norte e nordeste 5 .

o Estado do Maranhão apresentou coeficientes de prevalência e de detecção, respectivamente, de 7,7/10.000 e 6,8/10.000, ao final de 2006, segundo dados fornecidos pela Secretaria de Estado da Saúde.

No município de Buriticupu, o coeficiente de detecção de casos foi de 23,6/10.000 habitantes no ano 2000 e de 27,4/10.000

1. Centro de Referência em Doenças Infecciosas e Parasitárias, Departamento de Patologia, Universidade Federal do Maranhão, São Luis, MA.

Endereço para correspondência: Dr. Antonio Rafael da Silva. Dept ${ }^{\circ}$ de Patologia/UFMA. Praça Madre Deus 2, Térreo, Bairro Madre Deus, 65025-560 São Luis, MA.

e-mail: regionalsbmt@elo.com.br

Recebido para publicação em: 24/08/2007

Aceito em: 08/11/2007 
em 2001, o que o caracteriza como área hiperendêmica. Estes números são alarmantes e tenderiam a aumentar nos anos seguintes, visto que estão representando apenas a demanda espontânea aos serviços de saúde. A decisão de estudar a classe estudantil foi tomada levando em conta a situação descrita e tendo em mente o maior envolvimento das faixas etárias mais jovens nessa situação epidemiológica.

0 estudo foi desenvolvido com os objetivos de determinar o coeficiente de detecção da hanseníase na população escolar, identificar as formas clínicas, tratar os pacientes, examinar e tratar os comunicantes de escolares com hanseníase.

\section{MATERIAL E MÉTODOS}

Área de estudo. 0 município de Buriticupu localiza-se na pré-amazônia maranhense, entre as latitudes de $4^{\circ} \mathrm{S}$ e $5^{\circ} \mathrm{S}$ e longitude de $45^{\circ} 30^{\prime}$ e $47^{\circ}$ a oeste do Meridiano de Greenwich. Sua extensão territorial é de $2.719 \mathrm{~km}^{2}$, onde vivem 51.059 habitantes em 9.608 domicílios. 0 relevo é constituído por formações de tabuleiros, separados em faixas por drenos e grotões, situando-o a uma altitude de $200 \mathrm{~m}$ acima do nível do mar, guardando limites com os municípios de Bom Jardim, Alto Alegre do Pindaré, Santa Luzia, Arame, Amarante do Maranhão e Bom Jesus das Selvas. A economia sustenta-se num comércio bastante diversificado, destacando-se produtos madeireiros e agrícolas, como o arroz, o milho e a farinha além de uma pecuária de corte. Integrando a estrutura da rede de saúde possui dois hospitais, com 86 leitos e 10 unidades ambulatoriais.

A rede de ensino do município é integrada por 64 estabelecimentos, distribuídos em 7 áreas denominadas pólos, sendo que o pólo 1 corresponde à sede do município.

Da criação do município em 1997 até o ano 2000 foram registrados 531 casos de hanseníase, dos quais 334 (63\%) pertencentes ao sexo masculino e 197 (37\%), ao sexo feminino, com predomínio da faixa etária de 10 a 29 anos (42\%). As formas multibacilares (virchowiana e dimorfa) totalizaram $48,5 \%$ dos casos, enquanto as paucibacilares (indeterminada e tuberculóide), $51,5 \%$.

Detecção de casos. Foi empregado o método da busca ativa de casos, contando-se com a participação efetiva dos professores da rede de ensino público que, após receberem treinamento, fizeram uma triagem dos estudantes, identificando aqueles que apresentavam quaisquer alterações na pele. Em seguida, para a definição diagnóstica, foi realizado o exame clínico, pela equipe de pesquisa, de todos os selecionados na etapa anterior, sendo os dados anotados em fichas específicas.

0 diagnóstico de hanseníase foi definido com base na existência de alterações da coloração e/ou da sensibilidade ao exame clínico. Linfa cutânea foi coletada dos estudantes com lesões compatíveis com hanseníase, procedendo-se à coloração do material pelo método de Ziehl-Nielsen. A contagem dos bacilos seguiu a fórmula estabelecida por Ridley e Jopling?

Parte destes pacientes foi submetida à biópsia de pele, tomada na parte interna da borda da lesão, utilizando-se punch de $5 \mathrm{~mm}$ de diâmetro após assepsia e anestesia do local. 0 material biopsiado foi acondicionado em recipiente de plástico, contendo formol a $10 \%$ até o processamento laboratorial e leitura.

Para o cálculo do coeficiente de detecção considerou-se a população de estudantes regularmente matriculada nas escolas do município e que foi abrangida pela pesquisa.

0 tratamento foi feito com as drogas rifampicina, dapsona e/ou clofazimina, seguindo as recomendações do Ministério da Saúde ${ }^{3}$ e considerando a classificação operacional da Organização Mundial da Saúde 6 . A resposta ao tratamento, bem como o controle de cura, foi avaliada em exames clínicos periódicos e no acompanhamento da evolução das lesões.

Foram examinados todos os comunicantes dos casos novos, adotando-se os mesmos procedimentos descritos para 0 diagnóstico. Os comunicantes indenes foram encaminhados para a vacinação com BCG, seguindo as recomendações do Ministério da Saúde 3 .

\section{RESULTADOS}

Foram visitadas 53 das 64 escolas existentes no município, nos anos de 2004 e 2005, abrangendo-se uma população de 14.653 estudantes. A triagem, a encargo dos professores do município, resultou na seleção de 2.313 estudantes com quaisquer alterações na pele, que foram submetidos a exame clínico pela equipe de pesquisa. Foram detectados 20 casos de hanseníase, o que representa um coeficiente de detecção de 13,6/10.000 estudantes (Tabela 1). Deste total, 55,5\% eram do sexo masculino e $44,4 \%$ do sexo feminino, com idade entre 7 e 17 anos, destacando-se que 15 (75\%) pacientes eram menores de 15 anos. Dezoito (90\%) casos foram diagnosticados entre os estudantes das escolas da sede do município (pólo 1).

Em dezoito (90\%) pacientes havia alterações de pigmentação da pele (manchas hipocrômicas de tamanho variado, de bordas regulares ou não), infiltrações ou placas, acompanhadas de alteração da sensibilidade térmica e/ou dolorosa e/ou tátil. Houve predomínio de lesões nos membros inferiores, havendo acometimento de mais de um segmento corporal em seis pacientes. Em dois havia apenas área de anestesia. A baciloscopia foi positiva para bacilos álcoolácido resistentes em dois pacientes. Na definição da forma clínica, doze pacientes apresentaram a forma indeterminada; cinco, a tuberculóide e três, a dimorfa (Tabela 2).

0 exame histopatológico realizado em 11 (55\%) pacientes, dentre os quais os dois multibacilares, revelou achados comuns de hiperceratose, infiltrado inflamatório mononuclear em torno de vasos, anexos e nervos, assim como músculo eretor de pêlo isolado e vasos ectásicos.

Todos os pacientes receberam o tratamento específico. Dezenove (95\%) receberam alta por cura no serviço, não tendo apresentado estado reacional; um foi transferido para outro município pouco tempo após receber o diagnóstico.

$\mathrm{Na}$ avaliação dos comunicantes dos 20 pacientes foi diagnosticada a doença em um dos familiares, o qual recebeu 
Tabela 1 - Distribuição da população estudantil por escolas e doenças da pele, Buriticupu, Estado do Maranhão, em 2004 e 2005.

\begin{tabular}{lrrcrrrr}
\hline Pólo & $\begin{array}{c}\text { Escolas } \\
\mathrm{n}^{-}\end{array}$ & $\begin{array}{c}\text { Alunos } \\
\mathrm{n}^{-0}\end{array}$ & $\begin{array}{c}\text { Alunos triados pelos } \\
\text { professores* }\end{array}$ & Hanseníase & Ptiríase & Escabiose & Dermatofitose \\
\hline 1 & 8 & 7.785 & 1.172 & 18 & 431 & 160 & 37 \\
2 & 10 & 1.093 & 193 & 0 & 47 & 44 & 23 \\
3 & 8 & 591 & 109 & 0 & 47 & 17 & 5 \\
4 & 8 & 2.769 & 403 & 0 & 99 & 98 & 30 \\
5 & 5 & 466 & 106 & 0 & 25 & 24 & 9 \\
6 & 6 & 903 & 113 & 0 & 42 & 7 & 5 \\
7 & 8 & 1.046 & 217 & 2 & 102 & 11 & 10 \\
\hline Total & 53 & 14.653 & 2.313 & 20 & 793 & 361 & 119 \\
\hline
\end{tabular}

Pólo 1: sede do município, Pólos 2, 3, 4, 5, 6 e 7: área rural.

*condições diversas (cicatrizes, nevos, escoriações, despigmentação) foram identificadas em 1.020 destes estudantes.

Tabela 2 - Distribuição de casos de hanseníase segundo formas clínicas, Buriticupu, Estado do Maranbão, em 2004 e 2005.

\begin{tabular}{lcc}
\hline Forma clínica & Número & Percentagem \\
\hline Indeterminada & 12 & 60,0 \\
Tuberculóide & 5 & 25,0 \\
Dimorfa & 3 & 15,0 \\
\hline Total & 20 & 100,0 \\
\hline
\end{tabular}

tratamento e foi acompanhado pela equipe. Além disso, obteve-se história de hanseníase em outras oito pessoas que já tinham sido tratadas e recebido alta.

0 exame clínico dos 2.313 estudantes triados pelos professores permitiu, ainda, a identificação de outras doenças infecciosas da pele: pitiríase versicolor em 793, escabiose em 361 e dermatofitoses em 119. Condições diversas como cicatrizes, nevos, escoriações e despigmentações inespecíficas foram observadas em 1.020 destes estudantes (Tabela 3).

Tabela 3 - Distribuição dos casos de hanseníase e outras doenças infecciosas da pele por sexo e faixa etária, Buriticupu, Estado do Maranbão, em 2004 e 2005.

\begin{tabular}{|c|c|c|c|c|c|c|c|c|}
\hline \multicolumn{9}{|c|}{ Faixa etária } \\
\hline \multirow[t]{2}{*}{ (anos) } & \multicolumn{2}{|c|}{ Hanseníase } & \multicolumn{2}{|c|}{ Pitiríase versicolor } & \multicolumn{2}{|c|}{ Escabiose } & \multicolumn{2}{|c|}{ Dermatofitose } \\
\hline & M & $\mathrm{F}$ & $M$ & $\mathrm{~F}$ & M & $\mathrm{F}$ & M & $\mathrm{F}$ \\
\hline $0-6$ & 0 & 0 & 0 & 2 & 1 & 2 & 1 & 1 \\
\hline $7-10$ & 5 & 0 & 110 & 115 & 60 & 126 & 24 & 41 \\
\hline $11-14$ & 4 & 6 & 217 & 233 & 51 & 89 & 25 & 16 \\
\hline $15-17$ & 2 & 3 & 40 & 62 & 7 & 14 & 3 & 6 \\
\hline$>17$ & 0 & 0 & 6 & 10 & 4 & 7 & 1 & 1 \\
\hline \multirow[t]{2}{*}{ Total } & 11 & 9 & 373 & 420 & 123 & 238 & 54 & 65 \\
\hline & \multicolumn{2}{|c|}{20} & \multicolumn{2}{|c|}{793} & \multicolumn{2}{|c|}{361} & \multicolumn{2}{|c|}{119} \\
\hline
\end{tabular}

M: masculino, F: feminino.

\section{DISCUSSÃo}

Com a implantação da poliquimioterapia, a partir de 1981, os países endêmicos passaram a trabalhar com a possibilidade de eliminação da hanseníase ${ }^{4}{ }^{6}$. 0 controle efetivo da doença, no entanto, desafia a organização dos serviços de saúde e exige mobilização social, em função do longo período de incubação, do número elevado de casos, do estigma que a doença determina e das sequielas que parte dos pacientes pode desenvolver. Com esta consciência, buscou-se desenvolver ações integradas de controle, em parceria com as secretarias de saúde, de educação e de serviço social do município, estruturando-se o projeto intitulado "Controle da Hanseníase no município de Buriticupu - MA", com desenvolvimento em três fases: implantação (2004-2005), consolidação (2006-2007) e manutenção (2008-2010), com os objetivos de averiguar a situação epidemiológica da hanseníase no município, implantar ações descentralizadas de controle com a participação efetiva da escola e do Programa de Saúde da Família e estabelecer um sistema permanente de vigilância que permita a detecção e tratamento precoces de casos novos.

Os dados apresentados neste trabalho são o resultado de ações desenvolvidas na fase de implantação.

A busca ativa demonstrou ser um método eficaz na detecção de casos, tendo sido decisiva a participação dos professores na identificação dos estudantes com lesões cutâneas para a ampla cobertura que a pesquisa atingiu, além de ter tornado possível a setores diversos da sociedade maior esclarecimento sobre a doença e seus determinantes.

0 elevado coeficiente de detecção da doença entre os escolares, em baixas faixas etárias, corrobora a situação de hiperendemicidade do município, o que pode estar refletido na afirmação de Pinto Neto ${ }^{8}$ de que 0 deslocamento da incidência da hanseníase para menores de 15 anos indica um aumento na cadeia de transmissão do bacilo, assim como deficiência na vigilância e falha no controle da doença. A constatação de que 18 (90\%) casos diagnosticados são paucibacilares, sendo 12 da forma indeterminada, reforça a importância do diagnóstico e tratamento precoces, quando se considera a possibilidade de evolução para formas polares, potencialmente incapacitantes. De fato, não havia comprometimento importante de troncos nervosos e nem sinais de incapacidade física, no momento do diagnóstico, em nenhum dos vinte pacientes. Foi imprescindível fazer a classificação correta do caso, ao se diagnosticar a doença, a fim de que o paciente recebesse 0 tratamento adequado para obter a cura, diminuindo-se a transmissão da doença a partir dos multibacilares.

Chama a atenção a maior detecção de casos em escolas do pólo 1, localizadas na sede do município, ressaltando-se, ainda, neste contexto, o encontro de casos entre comunicantes de 
expressivo número de pacientes. Este fato poderia ser explicado pela aglomeração de pessoas, o que foi demonstrado em estudo das condições sócio-econômicas de 15 destes pacientes, o qual constatou que cerca de $60 \%$ das residências deles continham apenas três cômodos e eram habitadas por mais de cinco pessoas $^{10}$. Observação semelhante fora feita por outro autor, segundo o qual a hanseníase atinge principalmente populações de baixa renda e baixo nível sociocultural que vivem em aglomerados, onde a possibilidade de transmissão do bacilo é maior² .

A boa resposta terapêutica apresentada pelos pacientes corrobora, a nosso ver, a eficácia, reconhecida, da poliquimioterapia, enquanto a experiência de envolver o setor da educação no processo introduziu, na prática, um instrumento importante de controle da doença no município, tendo a vantagem de trazer para o conhecimento da sociedade os elementos atuantes na dinâmica de transmissão da doença.

\section{REFERÊNCIAS}

1. Araújo MG. Hanseníase no Brasil. Revista da Sociedade Brasileira de Medicina Tropical 36: 373-382, 2003.
2. Fonseca PHM, Cutrim RJCJ, Carneiro SFM. Hanseníase no Estado do Maranhão: análise de 5274 casos. Arquivo Brasileiro de Medicina 57:175-177,1983

3. Fundação Nacional de Saúde. Guia de Vigilância Epidemiológica. 5aa edição, Ministério da Saúde, Brasília, volume I, 2002.

4. Goulart IBM, Penna GO, Cunha G. Imunopatologia de Hanseníase a complexidade dos mecanismos da resposta imune do hospedeiro ao Mycobacterium leprae. Revista da Sociedade Brasileira de Medicina Tropical 35: 365-375, 2002.

5. Ministério da Saúde. Situação epidemiológica da hanseníase no Brasil. 2003. Disponível em http://www.portal.saude.gov.br/portal/arquivos. Acesso em 6 de junho de 2007.

6. Organização Mundial da Saúde. Guia para eliminação da Hanseníase como problema de Saúde Pública. 1ª edição, Geneve, 2000.

7. Organização Mundial da Saúde. Weekly Epidemilogical Record 13: 113-124, 2005

8. Pinto Neto JM, Villa TCS. Características epidemiológicas dos comunicantes de hanseníase que desenvolveram a doença, notificados no Centro de Saúde de Fernandópolis (1993 a 1997). Hansenologia Internationalis 24: 129136,1999 .

9. Ridley DS, Jopling WH. Leprosy Review 33: 119-128, 1962.

10. Silva Júnior CAD. Representação da Hanseníase pelas famílias dos estudantes do município de Buriticupu, acometidos pela doença. Monografia de conclusão de curso de graduação. Curso de Farmácia. Universidade Federal do Maranhão, São Luis, 2005. 